Introduction The aim of the present study was to evaluate the association between chronotype and age in day and rotating shift workers.

Methods The present cross-sectional study was carried out between October 2012 and February 2015 in a large German chemical company. Employees participating in a regular voluntary occupational health check-up were requested to complete a written questionnaire, which included items on sleeping behaviour extracted from the Munich Chronotype Questionnaire. Inclusion criteria comprised a fully completed questionnaire, not having used an alarm clock on free days, and being employed either as a day or rotating shift worker. Senior executive managers, trainees and interns were excluded. We used univariable and multivariable linear regression analyses to assess the association between age and chronotype (in minutes) in the whole sample and stratified by shift status.

Result Altogether 10348 persons completed the questionnaire, of which $4040(39.0 \%)$ met the inclusion criteria. Participants were on average 41.8 years old (SD: 10.2), mainly male (75.4\%) and engaged in day work (82.3\%). Mean chronotype was 03:23 (SD: $54 \mathrm{~min}$.) in the total sample, 03:16 (SD: 55 min.) in day and 03:57 (SD: 35 min.) in rotating shift workers. With increasing age, chronotype declined from 04:00 ( $\leq 29$ years) to $03: 08$ ( $\geq 50$ years) in the whole sample, and from 03:54 to $02: 59$ in day and $04: 25$ to $03: 45$ in rotating shift workers. Univariable and multivariable linear regression analyses correspondingly showed a significant decline of chronotype with age in both, day and rotating shift workers.

Discussion While day workers could benefit from a chronotype decrease, rotating shift workers could build up an intolerance regarding night work with increasing age. Shift workers might benefit from specific targeted prevention programs including sleep hygiene trainings.

\section{FREE OF CHOICE OPTIONS TO REDUCE SITTING: DOES IT WORK?}

$1,2 \mathrm{~V}$ Hermans*, ${ }^{3} \mathrm{~S}$ De Swerdt, ${ }^{4} \mathrm{~J}$ Seghers, ${ }^{1,5} \mathrm{~L}$ Godderis, $1,6 \mathrm{~L}$ Daenen. ${ }^{1}$ Knowledge,
Information and Research Center, IDEWE Group (External Service for Prevention and
Protection at Work), Leuven, Belgium; ${ }^{2}$ Department of Experimental and Applied
Psychology, Work and Organisational Psychology (WOPS), Faculty of Psychology and
Education Sciences Vrije Universiteit Brussel, Brussel, Belgium; ${ }^{3}$ Faculty of Economics and
Business, KU Leuven, Brussel, Belgium; ${ }^{4}$ Department of Kinesiology, Physical Activity, Sports
$\&$ Health Research Group, KU Leuven, Leuven, Belgium; ${ }^{5}$ Environment and Health, Faculty
of Medicine, KU Leuven, Louvain, Belgium; ${ }^{6}$ Department of Rehabilitation Sciences and
Physiotherapy, Human Physiology and Anatomy (KIMA), Faculty of Physical Education and
Physiotherapy, Vrije Universiteit Brussel, Brussels, Belgium

10.1136/oemed-2018-ICOHabstracts. 113

Introduction Increased cardiovascular diseases, obesity and diabetes are sedentary lifestyle. To tackle this problem, strategies to reduce sitting such as sit-stand tables. very low quality of evidence for the interventions. This study impact of a intervention reduc sitting

Methods An RCT-study was conducted in the office environment of a pharmaceutical company.: presentation of importance of good ergonomics/movement at the office and a check of the ergonomic set-up of the workstation. the experimental group (19) could use sit-stand tables, desk bikes and sit-stand chairs for 4 weeks. lso a weekly motivation email was sent. Sitting time was measured using ActivPAL ${ }^{\mathrm{TM}}$ accelerometers before and after the 4 weeks intervention both groups. Data were analysed using SPSS.
Results significant decrease in sitting time was the experimental group The reduction in sitting time was of the subjects attributed the reduction in sitting time to the use of sit-stand tables. of the desk bikes and sit-stand supports was. Half of the subjects revealed that these devices were no important contributors to reduced sitting.

Conclusion A decreased sitting time in the intervention group, subjectively mainly attributed to the use of sis important factors. this further.

\section{MUSCULOSKELETAL PAIN PREDICTS ILL-HEALTH RETIREMENT AMONG KOREAN WORKERS}

Se-Young Lee, Jun-Pyo Myong, HyoungRyoul Kim, Youn-Mo Cho, Mo-Yeol Kang*. Department of Preventive Medicine, College of Medicine, The Catholic University of Korea, Seoul, Korea

\subsection{6/oemed-2018-ICOHabstracts.114}

Introduction The aim of this study is to investigate the effect of musculoskeletal pain on IHR (ill-health retirement) among Korean workers.

Methods Data were collected from a sample from the first to fourth phases of the Korean Longitudinal Study of Ageing (KLoSA) which conducted in 2006, 2008, 2010, 2012, and 2014. IHR were defined as those who retired due to their health problems before their scheduled or regular retirement age in one of the follow-up surveys. Pain during the preceding month in multiple locations was combined into four sites (shoulder, upper extremities, low back, lower extremities). Hazard ratios (HR) of IHR were estimated by Cox regression, after adjusting for age, job category, annual income, history of disease (HTN and DM), BMI and smoking status.

Results The HR of IHR ( $n=477)$ was 1.49 (95\% confidence interval 1.01-2.20) for any site of pain among male workers, and 1.75 (1.23-2.49) among female workers. Risk of IHR was highest when $\mathrm{t}$ workers had upper extremities pain (Male, $\mathrm{HR}=2.71$; Female, $\mathrm{HR}=1.88$ ). Musculoskeletal pain in the other sites was also predictive of IHR. Subgroup analysis according to socioeconomic status showed that the association between musculoskeletal pain and IHR is greater in white collar worker and high income groups than the others (white collar, $\mathrm{HR}=2.15$; high income, $\mathrm{HR}=2.93$ ).

Conclusions Musculoskeletal pain independently predicted IHR. Moreover, our results revealed that socioeconomic status modified these effects of musculoskeletal pain on IHR. Employees with musculoskeletal pain may need specific support to maintain their work ability.

\section{WORKERS AGE 55 AND OLDER WORKING WITH PAIN}

1,2Marie-Christine Richard*, 1,2Marie-José Durand. 'Faculty of Medicine, Université de
Sherbrooke, Longueuil, Québec, Canada; ${ }^{2}$ Centre de recherche- Hôpital Charles-Le Moyne,
Longueuil, Québec, Canada

10.1136/oemed-2018-ICOHabstracts.115

Introduction The average worker in industrialised countries is ageing. The baby boomer generation (born 1946-1965) is gradually reaching retirement age. In Canada, the share of workers in the age $55+$ age group is expected to attain $24 \%$ in 2031. There is also a shortage of young workers to replace retirees. Although data suggest that at least one in five older workers suffers from chronic musculoskeletal pain, most will 